\title{
2-D solitary waves in thermal media with non-symmetric boundary conditions
}

\author{
By S.A. Louis ${ }^{1}$, T.R. Marchant ${ }^{1}$, and N.F. Smyth $h^{1,2}$
}

Optical solitary waves and their stability in focusing thermal optical media, such as lead glasses, are studied numerically and theoretically in $(2+1)$ dimensions. The optical medium is a square cell and mixed boundary conditions of Newton cooling and fixed temperature on different sides of the cell are used. Nonlinear thermal optical media have a refractive index which depends on temperature, so that heating from the optical beam and heat flow across the boundaries can change the refractive index of the medium. Solitary wave solutions are found numerically using the Newton conjugate gradient method, while their stability is studied using a linearised stability analysis and also via numerical simulations. It is found that the position of the solitary wave is dependent on the boundary conditions, with the centre of the beam moving toward the warmer boundaries, as the parameters are varied. The stability of the solitary waves depends on the symmetry of the boundary conditions and the amplitude of the solitary waves.

\section{Introduction}

Many different optical media have a refractive index that depends nonlinearly on the intensity of an imposed optical beam $[1,2,3]$. A balance between the nonlinear response and beam diffraction means that a bright

\footnotetext{
Address for correspondence: Prof. T. Marchant, School of Mathematics and Applied Statistics, University of Wollongong, Wollongong, 2522, N.S.W., Australia; email: tim_marchant@uow.edu.au

DOI: $10.1111 /(($ please add article doi $))$

(C) YYYY Wiley Periodicals, Inc., A Wiley Company

This is the author manuscript accepted for publication and has undergone full peer review but has not been through the copyediting, typesetting, pagination and proofreading process, which may lead to differences between this version and the Version of Record. Please cite this article as doi: 10.1111/sapm.12243

This article is protected by copyright. All rights reserved. 
optical solitary wave can form in a focusing medium and a dark solitary wave in a defocusing one. Such nonlinear optical media include nematic liquid crystals [2, 4], lead glasses and other thermal optical media [5, 6, 7] and photorefractive crystals [8,9], all of which are able to support the formation of solitary waves in this manner. For thermal optical media, the medium absorbs heat from the propagating beam, changing the refractive index and allowing solitary waves to be supported by a balance between the nonlinear refractive index change and diffraction $[6,7,10,11,12]$.

Experimental work on defocusing optical media (whose refractive index decreases with temperature) has shown that thermal media can support dark solitary waves [13]. This work further showed that an initial discontinuity in the intensity of the optical beam is smoothed by a dispersive shock wave whose trailing edge consists of dark solitary waves, which is expected as the governing equation is of nonlinear Schrödinger (NLS) type [14]. Conti et al. [15] performed experimental work on a defocussing thermal medium, describing the formation and evolution of dispersive shock waves from a gradient catastrophe. Of relevance to the present work, Alfassi et al. [16] considered the steering of beams in a thermal optical medium by applying different temperatures at the boundaries and found good agreement between numerical and observed solitary wave trajectories. Huang [17] numerically investigated the propagation of coupled dipole mode beams in a thermal optical medium, finding that the incoherent attraction of these component beams increases the stability of the composite structure. The stability enhancement is due to the broad nonlocal response of the medium, as for other nonlocal, nonlinear media $[18,19]$.

Optical solitary wave propagation in a nonlinear, nonlocal thermal medium is governed by a nonlinear Schrödinger (NLS)-type equation for the optical beam coupled with a Poisson equation for the temperature response of the medium. No exact solitary wave solutions have been found for this general system to date, only isolated solutions for fixed parameter values [20]. Hence, these systems have normally been analysed using approximate solution techniques, such as variational methods $[21,11]$, and modulation theory, in both nematic liquid crystals [11, 22] and colloidal media [23], finding good agreement with numerical solutions $[11,24,25]$ and experimental results [26, 27].

An alternative approach to finding full numerical solutions of the coupled thermal equation system is to search for stationary solutions

This article is protected by copyright. All rights reserved. 
via the separable solution method. This technique has been used to consider the interaction of multiple solitary waves governed by a range of different nonlocality conditions [28], as well as to consider the steady state behaviour of ground and excited state $(1+1)$-D solitary waves subject to a Newton cooling boundary condition [29]. Yang et al. [30, 31, 32 ] considered the stability and performance of numerical schemes for solving NLS-type equations, including the Newton conjugate-gradient method and imaginary time evolution method, while Wang [33] found that stable numerical solutions for single and coupled NLS equations are obtainable using a split-step finite difference method.

A nonlocal response is vital for the stability of $(2+1)$-D solitary waves governed by NLS-type equations $[4,5,34]$ as such waves are unstable in local media, displaying catastrophic collapse in a finite distance [1]. Yakimenko et al. [35] used a linear perturbation analysis to investigate the modulational stability of optical vortex solitons, finding that stable solutions are possible in a strongly nonlocal medium. Elan et al. [36] demonstrated that for NLS-type equations, solitary waves are unstable if there are negative eigenvalues of the fourth order linearised operator governing small perturbations of the solitary wave. They also showed that the nature of the instability could be inferred from the eigenvectors corresponding to the negative eigenvalues, with symmetric eigenvectors indicative of an amplitude instability and asymmetric eigenvectors indicating a drift instability.

In this paper, the $(2+1)$-D system governing nonlinear optical beam propagation in thermal media, comprising an NLS-type equation for the optical field and a Poisson equation for the temperature of the medium, is studied. Non-symmetric boundary conditions are applied at one or more of the boundaries of a square cell geometry. In Section 2 the governing equations are outlined and the convergence rates of the Newton conjugate-gradient and imaginary time evolution numerical methods are compared. In Section 3 numerical solutions of the governing equations are generated for various boundary configurations. It is found that the position of the solitary wave within the cell can be easily changed by adjusting the boundary conditions, with the solitary wave migrating towards the warmer boundaries. Section 4 studies the stability of the solitary waves analytically using power-propagation constant curves and a linear spectral stability analysis, as well as via numerical solutions of the full thermal governing equations. The numerical and analytical stability analyses are found to be in agreement. Both predict that the

This article is protected by copyright. All rights reserved. 
stability of a solitary wave strongly depends on the boundary conditions and the amplitude of the solitary wave.

\section{Governing equations}

Let us consider the propagation of an input Gaussian, coherent, polarised light beam of wavelength $\lambda$, wavenumber $k=2 \pi / \lambda$, power $P_{0}$ and width $W_{B}$ through a nonlinear optical medium whose refractive index depends linearly on temperature change, so that $\Delta n=\beta \Delta T$, where $\Delta n$ is the change in refractive index due to a temperature change $\Delta T[6]$. Let us take the $Z$ direction to be the direction of propagation of the beam and the $X$ coordinate the direction of polarisation of the beam, with $Y$ completing the coordinate triad. The electric field of the optical beam in the polarisation direction will be denoted by $E$. In the slowly varying, paraxial approximation, the dimensional equations governing the propagation of the beam are then $[4,6,7]$

$$
2 i k \frac{\partial E_{x}}{\partial Z}+\nabla^{2} E_{x}+2 k^{2} \frac{\Delta n}{n_{0}} E_{x}=0, \quad \kappa \nabla^{2} \Delta T=-\alpha\left|E_{x}\right|^{2} .
$$

The Laplacian $\nabla^{2}$ is in the transverse plane $(X, Y)$ to the propagation direction $Z$. The parameter $\kappa$ is the thermal conductivity of the medium, $n_{0}$ is the linear refractive index and $\alpha$ is the thermal absorption coefficient. This system of equations can be set in non-dimensional form via the variable transformations

$$
X=W x, \quad Y=W y, \quad Z=C_{z} z, \quad E_{x}=C_{e} E, \quad \Delta T=C_{T} \theta,
$$

where

$$
W^{2}=\frac{2 n_{0}}{\beta C_{T} k^{2}}, \quad C_{z}=\frac{2 n_{0}}{\beta C_{T} k}, \quad C_{e}^{2}=\frac{2}{\pi W_{B}^{2}} P_{0}
$$

and $C_{T}$ is a typical temperature change. The non-dimensional system of equations governing the propagation of the optical beam in the thermal medium is then

$$
\imath \frac{\partial E}{\partial z}+\frac{1}{2} \nabla^{2} E+2 E \theta=0, \quad \nu \nabla^{2} \theta+2|E|^{2}=0,
$$

with the non-dimensional thermal conductivity

$$
\nu=\frac{\pi K C_{T}}{\alpha P_{0}}\left(\frac{W_{B}}{W}\right)^{2} .
$$

This article is protected by copyright. All rights reserved. 
Typical parameter values are $C_{T}=10 K, n_{0}=1.8, \kappa=0.7 \mathrm{~W} /(\mathrm{mK})$, $\beta=14 \times 10^{-6} \mathrm{~K}^{-1}, \alpha=1 \mathrm{~m}^{-1}, P_{0}=1 \mathrm{~W}, \lambda=488 \mathrm{~nm}$ and $W_{B}=$ $50 \mu \mathrm{m}$ for lead glass [6]. These values give a non-dimensional thermal conductivity of $\nu=354$. The non-dimensional thermal conductivity is then large, $O(100)$, so that the optical response of the thermal medium is termed nonlocal $[2,4]$. This nonlocal response is vital as it stabilises $(2+1)$-D solitary waves propagating in the thermal medium, noting that $(2+1)$-D solitary waves are unstable in local media [1]. Equation (4) also governs the propagation of an optical beam in a nematic liquid crystal $[2,4]$. In this case $\theta$ is the rotation of the nematic molecules due to the optical beam above the rest angle in the absence of the optical field. Furthermore, similar systems of equations to (4) apply to many nonlinear optical medium with a diffusive response [3].

For simplicity, we take the thermal medium to be a square cell of side length $L$. To investigate the effects of non-symmetric boundary conditions on the propagation of solitary waves in this nonlinear, nonlocal thermal medium, we consider differential heating at the medium boundaries. A Dirichlet boundary condition is applied to some of the boundaries, while a mixed boundary condition is applied at the other boundaries. For the optical field the usual assumption of reflective Dirichlet boundary conditions is made, so that the beam is confined to the cell and there is no (optical) electric field outside of it [24, 37, 38]. The usual dielectric boundary conditions that the parallel component of the electric field $E$ and the normal component of the electric displacement $D$ are continuous give that $E=0$ on all boundaries. While in experiments there is some leakage from the cell, the inclusion of this external field would be a non-trivial exercise, worthy of a future study. The full boundary conditions are then

$$
\begin{aligned}
& E=0 \text { at } x= \pm \frac{L}{2}, y= \pm \frac{L}{2}, \\
& \theta=0 \text { at } x=\frac{L}{2}, y=\frac{L}{2}, \\
& \theta_{x}-\beta_{x}\left(\theta-\theta_{B, x}\right)=0 \text { at } x=-\frac{L}{2}, \\
& \theta_{y}-\beta_{y}\left(\theta-\theta_{B, y}\right)=0 \text { at } y=-\frac{L}{2} .
\end{aligned}
$$

Here, $\beta_{x}$ and $\beta_{y}$ are the Biot numbers and $\theta_{B, x}$ and $\theta_{B, y}$ are the ambient temperatures at the relevant cell boundaries. The medium geometry

This article is protected by copyright. All rights reserved. 


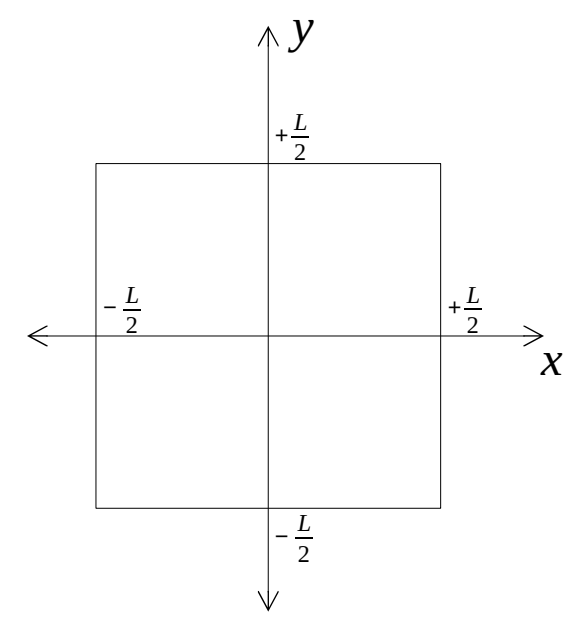

Figure 1. Schematic showing the thermal medium geometry and boundaries.

is show in Figure 1. The mixed condition represents Newton cooling, with $\beta_{x}=\beta_{y}=0$ corresponding to perfectly insulated boundaries and $\beta_{x}, \beta_{y} \rightarrow \infty$ to fixed temperature boundary conditions $\theta=\theta_{B, x}$ and $\theta=\theta_{B, y}$

To find solitary wave solutions of the thermal equations and boundary conditions (4)-(9), we seek a travelling wave solution of the form $E(x, y, z)=u(x, y) e^{i \mu z}$, where $u$ is real and $\mu$ is the propagation constant. The thermal equations (4) then reduce to

$$
\frac{1}{2} \nabla^{2} u+2 u \theta-\mu u=0, \quad \nu \nabla^{2} \theta+2 u^{2}=0,
$$

with the boundary conditions

$$
u=0 \quad \text { at } \quad x= \pm \frac{L}{2}, \quad y= \pm \frac{L}{2}
$$

and the boundary conditions for $\theta$ are as in (7)-(9).

To solve the thermal system (7)-(11) we tested two numerical methods for convergence and speed, the Newton Conjugate-Gradient (Newton-CG) method and the Imaginary Time Evolution Method (ITEM) $[30,31,32]$. Figure 2 shows the maximum error, $\epsilon$, between

This article is protected by copyright. All rights reserved. 
each iteration versus the iteration number, $n$, for each method. Both numerical schemes converge with a comparable number of iterations when using a stopping condition of $\epsilon=10^{-10}$. However, each iteration of the Newton-CG method was significantly faster, so we used this method throughout the solution space for the thermal system. A detailed description of the Newton-CG method is given in Appendix A. The computation time required to solve the case for which $\theta=0$ at all four boundaries was approximately 53 minutes with the ITEM method using a desktop PC with dual Intel Core i5-2500K processors and 8GB of RAM. This is about two orders of magnitude slower than the NewtonCG method, which reached the solution in 20 seconds.

\section{Results and discussion}

In all the examples considered we used a cell of length $L=30$ and choose $\Delta x=\Delta y=0.682$. The large thermal conductivity limit is also considered, with $\nu=100$.

\subsection{Single boundary with non-zero ambient temperature}

In this section we consider the behaviour of the solitary wave solution as the temperature on the $y=-L / 2$ cell boundary changes. The other boundaries are fixed at the ambient temperature. The boundary conditions used are (11) for $u$, but with $\beta_{x} \rightarrow \infty$, and $\theta_{B, x}=0$ so that

$$
\theta=0, \quad x= \pm \frac{L}{2}, \quad y=\frac{L}{2} ; \quad \theta_{y}-\beta_{y}\left(\theta-\theta_{B, y}\right)=0, \quad y=-\frac{L}{2} .
$$

Figure 3 shows thermal solitary wave solutions of (10) with the boundary condition (12) for one large heat loss boundary and three boundaries at ambient temperature. Shown are contour plots of the electric field amplitude, $u$, and temperature $\theta$ in the $(x, y)$ plane. The three solitary waves are for $\theta_{B, y}=0,0.2$, and 0.6. The other parameters are $\beta_{y}=100$ and $\mu=1$. When $\theta_{B, y}=0$ all four of the cell boundaries are at the same temperature and the solitary wave is symmetric, with the peak of the solitary wave, of amplitude $a=2.86$, located at the centre of the cell, $(x, y)=(0,0)$. As the ambient temperature $\theta_{B, y}$ of the warm cell boundary increases, the peak of the solitary wave decreases in amplitude and moves towards the warmer boundary. For the $\theta_{B, y}=0.2$ case the peak amplitude has decreased to $a=2.65$ and is located at

This article is protected by copyright. All rights reserved. 


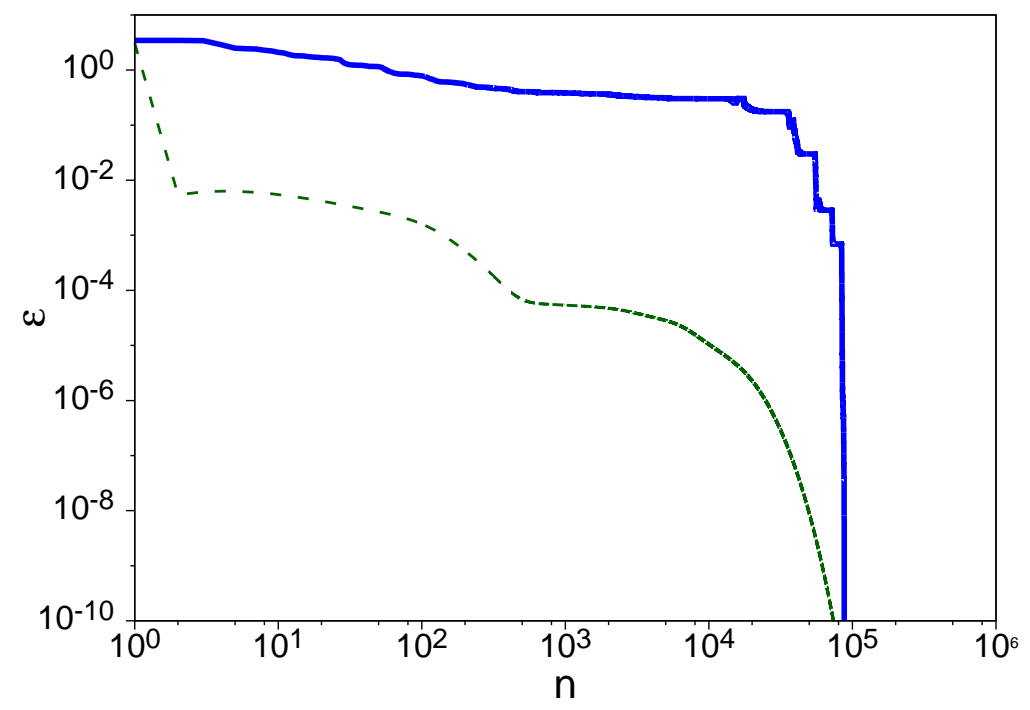

Figure 2. (Colour online) Comparison of convergence rates for the numerical schemes, with the maximum error between each iteration and the numerical solution, $\epsilon$, versus the number of iterations, $n$. NewtonCG method: solid (blue) line; ITEM method: dashed (green) line.

$(x, y)=(0,-7.0)$. For $\theta_{B, y}=0.6$ the amplitude has decreased further to $a=1.35$, while the wave peak has migrated towards the warmer boundary and is located at $(x, y)=(0,-12.2)$.

Figure 4 shows a 1-D section of the 2-D solitary waves shown in Figure 3 taken along $x=0$ through the centre of the cell. The behaviour of the solitary wave is qualitatively similar to the $(1+1)$-D solitary waves in Figure 4 of [29], but there are some differences. The temperature profile is not linear away from the solitary wave peak in the 2-D case, due to the 2-D heat diffusion, and higher values of $\theta_{B, y}$ are needed at the boundary to achieve the same offset from a centred wave position. This difference between the $(1+1)$ - $\mathrm{D}$ and $(2+1)$-D solitary waves is due to the Green's function of the heat equation in (4) being linear in $(1+1)$-D and logarithmic in $(2+1)$-D.

The peak values of $u$ and $\theta$ are co-located for small values of the boundary temperature $\theta_{B, y}$. However, for large values of $\theta_{B, y}$ this is not

This article is protected by copyright. All rights reserved. 

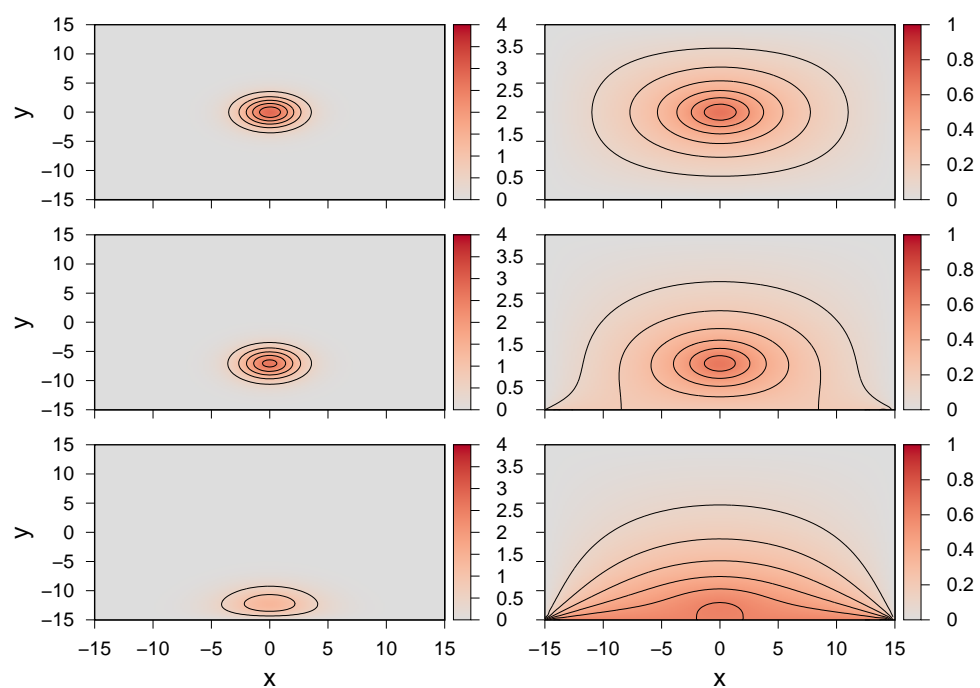

Figure 3. (Colour online) Thermal solitary waves, large $\beta$ case. Electric field amplitude, $u$ (left column), and temperature, $\theta$ (right column), in the $(x, y)$ plane. $\theta_{B, y}=0$ : top row; $\theta_{B, y}=0.2$ : middle row; $\theta_{B, y}=0.6$ : bottom row. Contours are positive values at intervals of 0.5 for $u$ and at intervals of 0.1 for $\theta$. The parameters $\beta_{y}=100$ and $\mu=1$.

the case. It may also be noted that for small values of $\theta_{B, y}$ the gradient of $\theta$ is positive at the warm cell boundary, indicating heat flow from the cell. For the $\theta_{B, y}=0.6$ case the gradient is nearly zero. For small $\theta_{B, y}$ the electric field is large, causing significant internal heating of the medium, with the heat lost at the cell boundary. For the $\theta_{B, y}=0.6$ case the electric field and the internal heating are smaller, leading to near equilibrium between the medium and the warm cell boundary.

Figures 5 and 6 show thermal wave solitary solutions of (10) with a boundary condition (12) with low heat loss $\beta_{y}=0.2$. In Figure 5 contour plots of the electric field $u$ and temperature $\theta$ are shown in the $(x, y)$ plane. The three solitary waves are for $\theta_{B, y}=0,0.2$ and 0.6 . The other parameters are the same as in Figure 3, except for the Biot number at the mixed boundary, which is small. In this case when $\theta_{B, y}=0$ the steady state temperature at the left hand cell boundary is $\theta(-L / 2)=0.10$,

This article is protected by copyright. All rights reserved. 


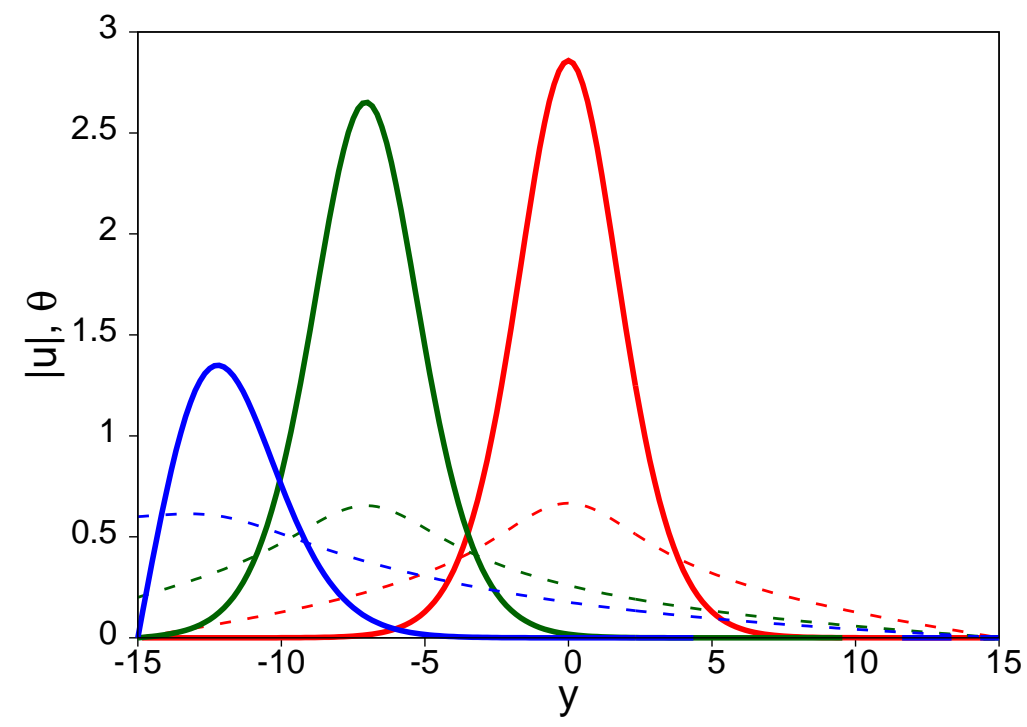

Figure 4. (Colour online) Thermal solitary wave cross sections through $x=0$, large $\beta$ case. Shown are the electric field, $u$ (solid), and temperature, $\theta$ (dashed). $\theta_{B, y}=0$ : red line, centre; $\theta_{B, y}=0.2$ : green line, slightly offset; $\theta_{B, y}=0.6$ : blue line, near boundary. The other parameters are $\beta_{y}=100$ and $\mu=1$.

while the peak of the solitary wave, of amplitude $a=2.77$, is located at $(x, y)=(0,-2.83)$. This wave shows a number of differences compared with the $\theta_{B, y}=0$ large Biot number case shown in Figure 3 . The wave is non-symmetric, being located closer to the warm cell boundary, and the amplitude is slightly lower. As $\theta_{B, y}$ increases the temperature at the warm cell boundary increases and approaches $\theta_{B, y}$, and, as for the large Biot number case, the solitary waves decrease in amplitude and migrate closer to the warm cell boundary. For the $\theta_{B, y}=0.2$ case the wave amplitude is $a=2.58$ and the wave is located at $(x, y)=(0,-11.0)$. For the $\theta_{B, y}=0.6$ case the amplitude has decreased to $a=1.80$ with the peak location $(x, y)=(0,-12.3)$ close to the cell boundary.

Figure 7 shows the location of the thermal solitary wave peak, $\zeta_{y}$, along the $x=0$ cell axis versus the ambient temperature $\theta_{B, y}$, with $\mu=1$. Shown are curves corresponding to $\beta_{y}=0.2,0.6$ and 100 , with the $\beta_{y}=100$ case very close to the result obtained in the large Biot num-

This article is protected by copyright. All rights reserved. 

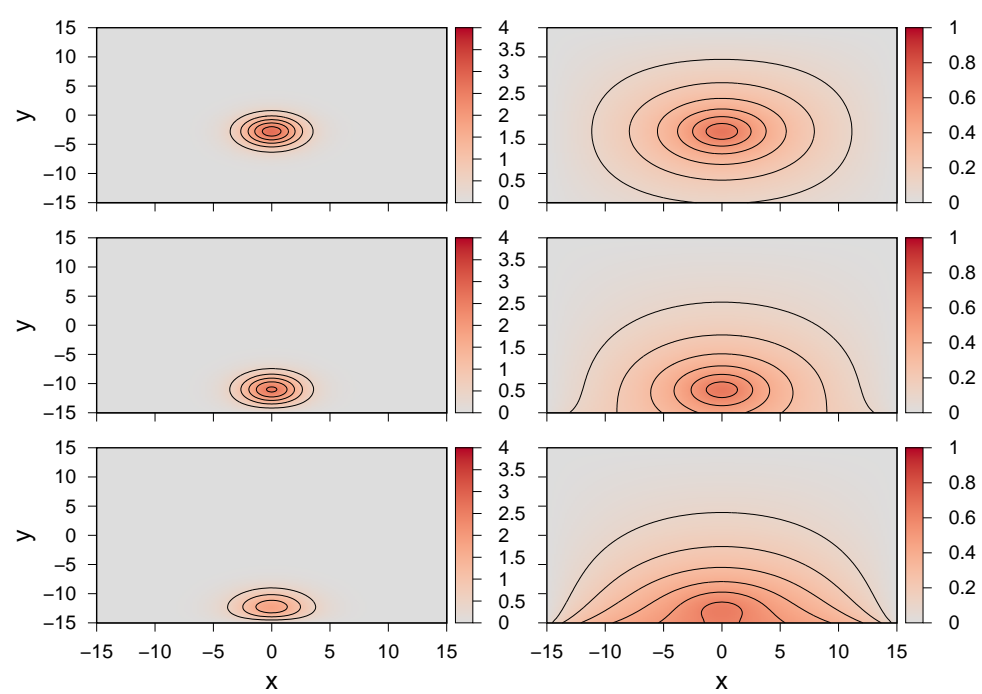

Figure 5. (Colour online) Thermal solitary waves, small $\beta$ case. Shown are the electric field amplitude, $u$ (left column), and temperature, $\theta$ (right column). $\theta_{B, y}=0$ : top row; $\theta_{B, y}=0.2$ : middle row; $\theta_{B, y}=0.6$ : bottom row. Contours are positive values at intervals of 0.5 for the $u$ figures, and values at intervals of 0.1 for the $\theta$ figures. The other parameters are $\beta_{y}=0.2$ and $\mu=1$.

ber limit. As the ambient temperature at the cell boundary is increased, the thermal solitary wave migrates towards the warmer boundary, approaching $\zeta_{y} \approx-12.7$. Increasing the ambient temperature causes the amplitude of the solitary wave to decrease to zero, suggesting that no solitary wave solution exists once the thermal gradient between the cell boundaries becomes too large. For a given ambient temperature at the mixed boundary, decreasing the Biot number also causes the thermal solitary wave to shift towards the warmer boundary. These results are qualitatively similar to the $(1+1)$-D solitary wave case of [29], with both showing that the solitary wave positions approach a location at around $80 \%$ of the distance to the cell boundary. However, the migration towards the boundary is faster with respect to increasing $\theta_{B, y}$ in the $(2+1)$-D case.

This article is protected by copyright. All rights reserved. 


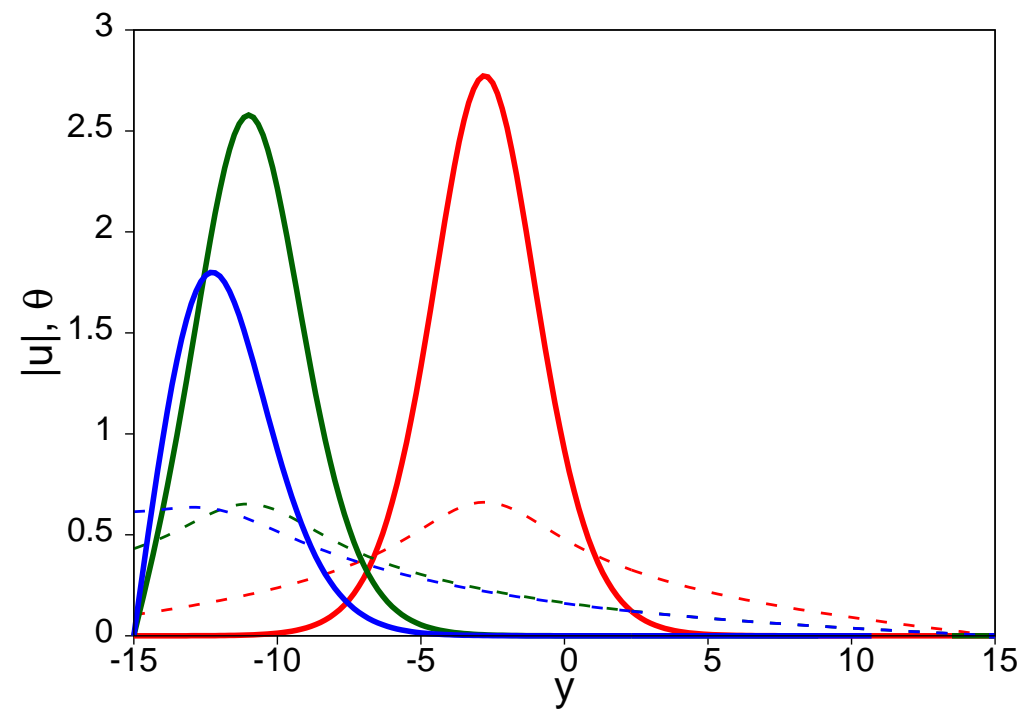

Figure 6. (Colour online) Thermal solitary waves, small $\beta$ case. Shown are the electric field amplitude, $u$ (solid), and temperature, $\theta$ (dashed), versus y. $\theta_{B, y}=0$ : red line (centre); $\theta_{B, y}=0.2$ : green, slightly offset line; $\theta_{B, y}=0.6$ : blue line, near boundary). The other parameters are $\beta_{y}=0.2$ and $\mu=1$.

Figure 8 shows the amplitude of the thermal solitary wave, $a$, as a function of the ambient temperature, $\theta_{B, y}$, for $\mu=1$. The curves correspond to $\beta_{y}=0.2,0.6$ and 100. For a given choice of Biot number, the solitary wave amplitude decreases as $\theta_{B, y}$ increases. The solitary wave amplitude decreases to $a \approx 0$ at a given value of $\theta_{B, y}$, after which thermal solitary waves do not exist. This threshold value of $\theta_{B, y}$ decreases as the Biot number increases, in particular it decreases from $\theta_{B, y}=1.04$ for $\beta_{y}=0.2$ to $\theta_{B, y}=0.71$ for $\beta_{y}=10$. Hence, if the heat loss rate at the Newton cooling boundary is too great, then a solitary wave cannot exist.

Figure 9 shows first excited state solitary wave solutions of (10) and (12). Shown are contour plots of the electric field $u$ and the temperature $\theta$ in the $(x, y)$ plane. The three solitary waves are for $\theta_{B, y}=0,0.1$ and 0.2 . The other parameters are $\beta_{y}=100$ and $\mu=0.1$. As $\theta_{B, y}$ increases, the structure of the electric field, $u$, changes. For $\theta_{B, y}=0$ the solitary

This article is protected by copyright. All rights reserved. 


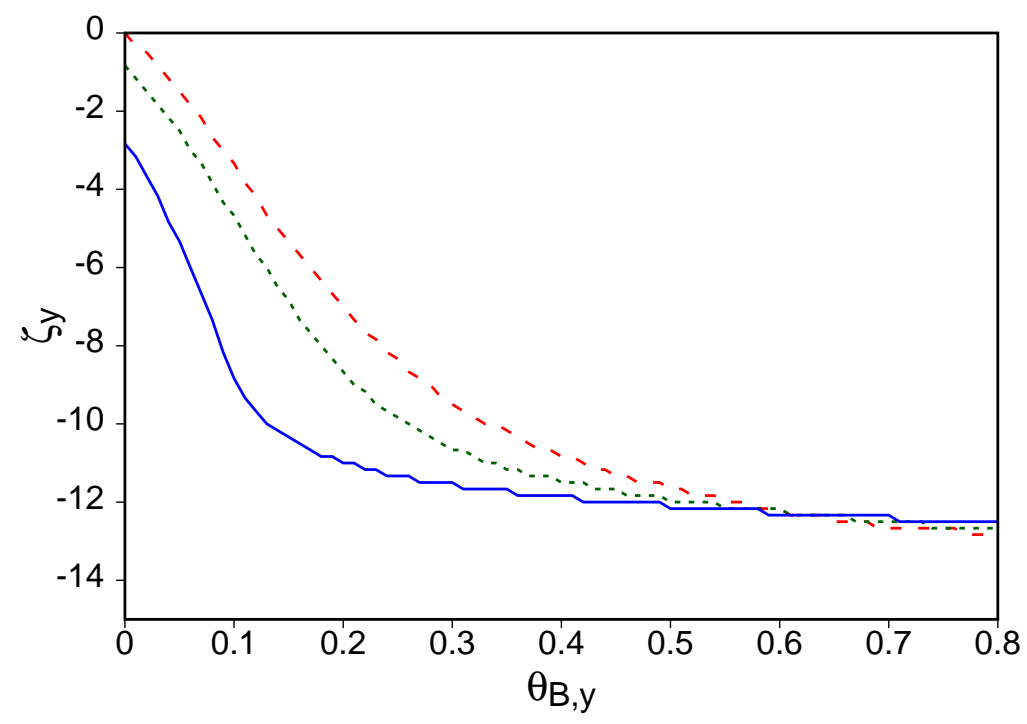

Figure 7. (Colour online) Location of the peak of thermal solitary wave, $\zeta_{y}$, versus the ambient temperature, $\theta_{B, y}$, for $\mu=1$. $\beta_{y}=0.2$ : solid (dark blue) line; $\beta_{y}=0.6$ : dotted (green) line; $\beta_{y}=100$ dashed (red) line. The edge of the domain is $y=-15$.

wave is symmetric, with a single maximum $a=1.11$ at $(x, y)=(0,0)$ and an annular shaped minima at $a=-0.50$ at a radial distance of $\zeta_{r}=0.65$ from the centre of the cell. For $\theta_{B, y}=0.1$ the solitary wave has a maximum of $a=0.94$ at $(x, y)=(0,-5.17)$, with three discrete minima. One of the minima is on the $x=0$ axis, with $a=-0.55$ at $y=2.17$, while the other two minima both have $a=-0.62$ at locations $(x, y)=( \pm 6.17,-9.0)$. For the $\theta_{B, y}=0.2$ case the maximum with $a=$ 0.45 is pushed closer to the boundary, at $(x, y)=(0,-6.5)$. There are now two discrete minima, both lying on the $x=0$ axis, with $a=-0.68$ at $(x, y)=(0,0.83)$ and $a=-0.60$ at $(x, y)=(0,-12.17)$. In contrast to the electric field, in all cases the temperature response $\theta$ has a single maximum. This is a result of the nonlocal heat diffusion, which smooths out the temperature response for large $\nu$. Figure 10 shows cross sections through $x=0$ of the 2-D solitary waves shown in Figure 9. For the cases where $\theta_{B, y}=0$ and $\theta_{B, y}=0.2$ the slice along the $x=0$ axis passes through the local maxima and minima in $(x, y)$ space, so the maximum

This article is protected by copyright. All rights reserved. 


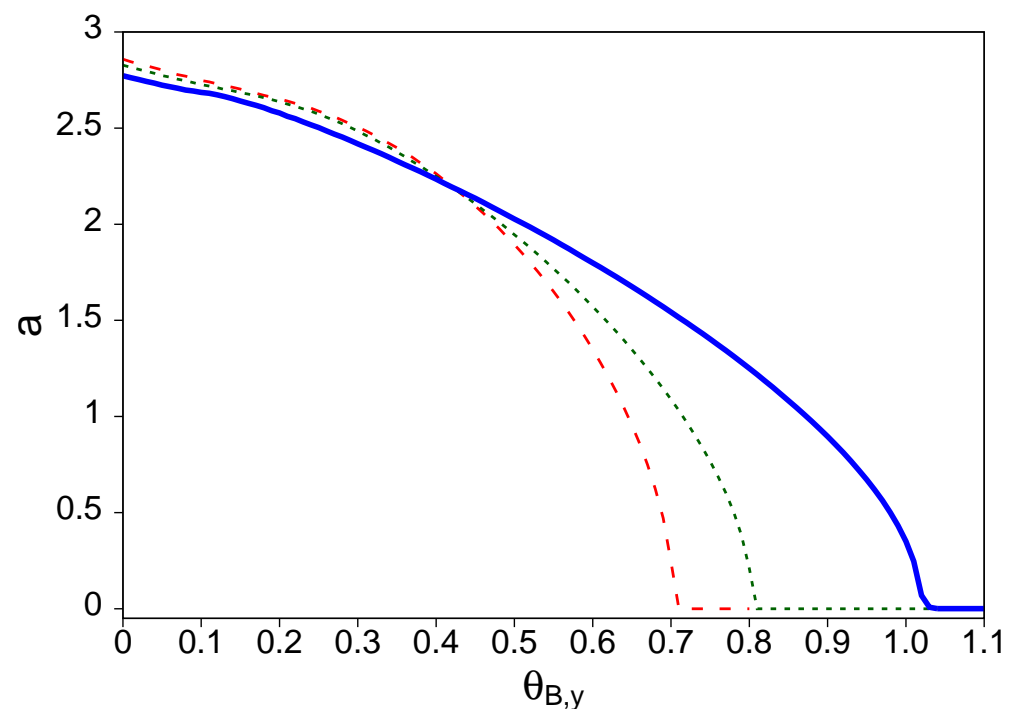

Figure 8. (Colour online) Amplitude of the thermal solitary wave, $a$, versus the ambient temperature, $\theta_{B, y}$, for $\mu=1$. $\beta_{y}=0.2$ : solid (dark blue) line; $\beta_{y}=0.6$ : dotted (green) line; $\beta_{y}=100$ : dashed (red) line.

and minima of Figure 10 are as in Figure 9. For the $\theta_{B, y}=0.1$ case, the maximum at $y=-5.17$ and minimum at $y=2.17$ both correspond to local extrema in Figure 9, while the minimum $a=-0.215$ at $y=11.83$ does not.

\subsection{Two adjacent boundaries with non-zero ambient temperature}

In this section we consider the scenario of two adjacent sides with a mixed boundary condition, $x=y=-L / 2$, with the other two boundary conditions at the ambient temperature. The boundary conditions for $\theta$ are

$$
\begin{array}{r}
\theta=0, \text { at } x=\frac{L}{2}, \quad y=\frac{L}{2}, \\
\theta_{x}-\beta_{x}\left(\theta-\theta_{B, x}\right)=0, \text { at } x=-\frac{L}{2}, \\
\theta_{y}-\beta_{y}\left(\theta-\theta_{B, y}\right)=0, \text { at } y=-\frac{L}{2} .
\end{array}
$$

This article is protected by copyright. All rights reserved. 


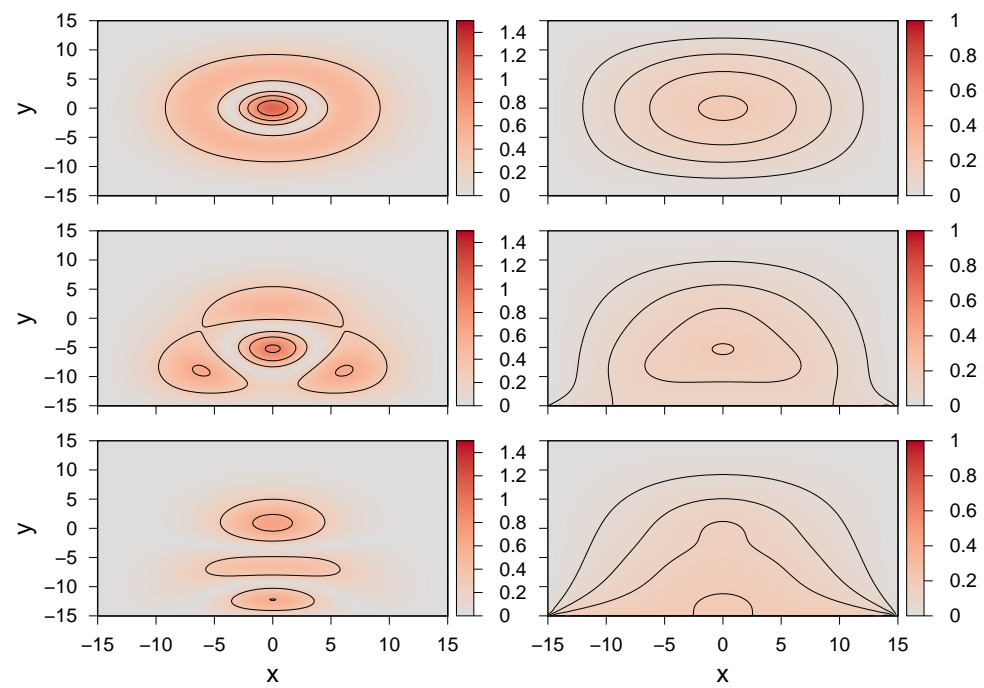

Figure 9. (Colour online) Thermal solitary waves, first excited state. $u$ (left column) and $\theta$ (right column). $\theta_{B, y}=0$ : top row; $\theta_{B, y}=0.1$ : middle row; $\theta_{B, y}=0.2$ : bottom row. Contours are values at intervals of 0.3 for $u$ and at intervals of 0.05 for $\theta$. The other parameters are $\beta_{y}=100$ and $\mu=0.1$.

Figure 11 shows two thermal solitary wave cases with Newton cooling on two adjacent boundaries. Shown are the electric field, $u$ and temperature, $\theta$ (right column) for a ground state solitary wave with $\theta_{B, y}=0.5, \theta_{B, x}=0.3$ and $\mu=1$, and for the first excited state solitary wave with $\theta_{B, y}=0.2, \theta_{B, x}=0.1$ and $\mu=0.1$. The other parameters are $\beta_{x}=\beta_{y}=100$. This choice of boundary conditions pushes the peak of the thermal solitary wave towards the corner of the cell at $(x, y)=(-15,-15)$. The peak amplitude is $a=1.45$ at $(x, y)=(-4.17,-11.17)$, which is almost, but not quite, co-located with the maximum cell temperature $\theta_{\max }=0.59$. For the first excited state the structure of the electric field, $u$, is complicated, with two main local maxima and two local minima. The main solitary wave maximum of $a=4.61 \times 10^{-4}$ is at $(x, y)=(-2.0,-7.5)$, with another maximum $a=2.2 \times 10^{-4}$ at $(x, y)=(-10.3,6.7)$. The minima are $a=-4.11 \times 10^{-4}$ at $(x, y)=(0.17,-0.5)$ and $a=-5.36 \times 10^{-4}$ at $(x, y)=(-3.0,-12.67)$.

This article is protected by copyright. All rights reserved. 

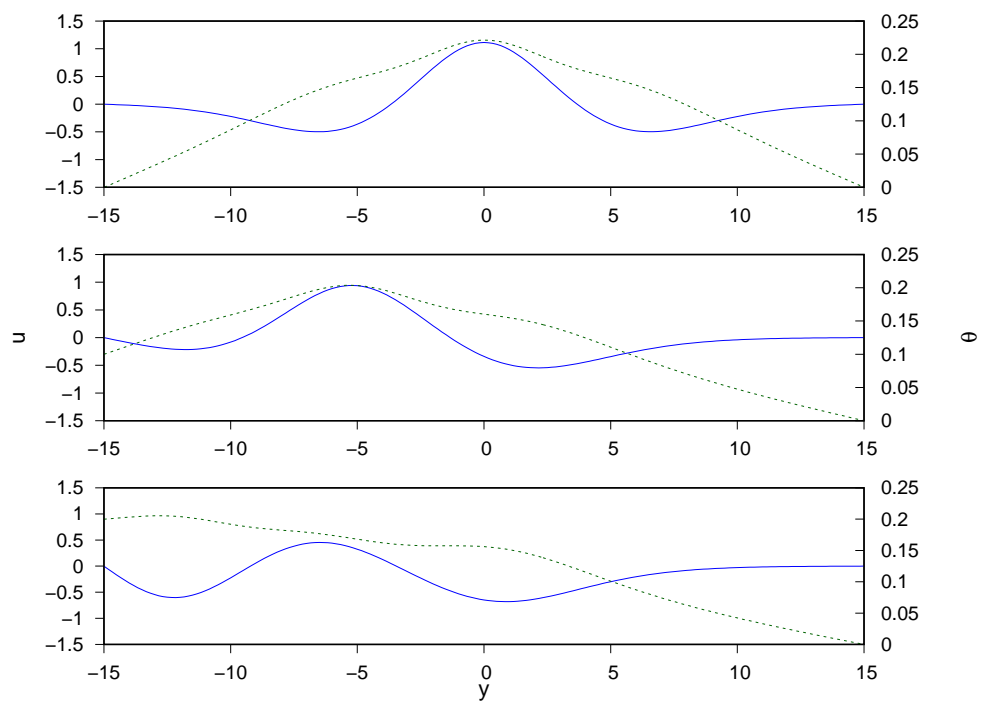

Figure 10. (Colour online) Excited state thermal solitary wave cross sections. Shown are the electric field amplitude, $u$ (solid), and temperature, $\theta$ (dashed), versus $y$. Note that the scales for $u$ (lhs) and $\theta$ (rhs) are different. $\theta_{B, y}=0$ : red line, centre; $\theta_{B, y}=0.1$ green line, slightly offset; $\theta_{B, y}=0.2$ blue line, near boundary. The other parameters are $\beta_{y}=100$ and $\mu=0.1$.

As with the excited state solitary wave solutions of Figure 9, the temperature response $\theta$ has a single maximum as a result of the large thermal conductivity $\nu$.

Figure 12 shows the amplitude, $a$, of the ground state thermal solitary wave in $\left(\theta_{B, x}, \theta_{B, y}\right)$ parameter space, while Figure 13 shows the position of the ground state thermal solitary wave in the $\left(\theta_{B, x}, \theta_{B, y}\right)$ parameter space, with offset along the $x$ axis $\left(\zeta_{x}\right)$ and offset along the $y$ axis $\left(\zeta_{y}\right)$. The other parameters are $\beta_{x}=\beta_{y}=100$. As expected, increasing $\theta_{B, x}$ or $\theta_{B, y}$ causes the amplitude of the solitary wave to decrease, as well causing its peak to migrate towards the respective cell boundaries. The solitary wave response is symmetric about the line $\theta_{B, x}=\theta_{B, y}$, but the value of $r=\sqrt{\theta_{B, x}^{2}+\theta_{B, y}^{2}}$ at which the solitary wave amplitude $a$ reaches zero is not constant. The values of $r$ at which $a=0$ range from $r=0.71$ along the $\theta_{B, x}=0$ and $\theta_{B, y}=0$ axes in Figure 12 to $r=0.83$ 

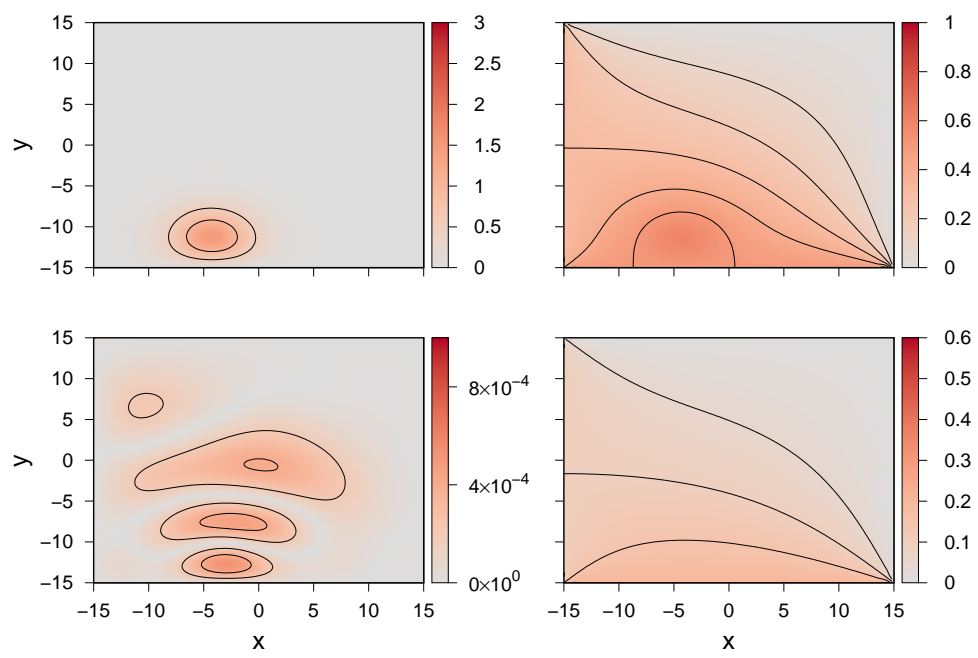

Figure 11. (Colour online) Thermal solitary waves with Newton cooling on two adjacent boundaries. Shown are the electric field amplitude, $u$ (left column), and temperature, $\theta$ (right column). Top row: ground state solitary wave with $\theta_{B, y}=0.5$ and $\theta_{B, x}=0.3$ and $\mu=1$. Bottom row: first excited state solitary wave with $\theta_{B, y}=0.2$ and $\theta_{B, x}=0.1$ with $\mu=0.1$. The other parameters are $\beta_{x}=\beta_{y}=100$. Contours are at intervals of 0.5 for the top left figure, 0.1 for top right, $2 \times 10^{-4}$ for the bottom left and 0.05 for the bottom right.

for $\theta_{B, x}=\theta_{B, y}=0.59$, with the difference in these values a result of the square cell geometry.

\section{Solitary wave stability}

All the results presented in this section are for the case of a single cell boundary with non-zero ambient temperature, as described at the start of section $\S 3.1$ with boundary condition (12). All examples presented in this section use the parameter $\beta_{y}=100$. So the presented results explore the effect of the boundary temperature $\theta_{B, y}$ and the propagation parameter $\mu$ on stability.

This article is protected by copyright. All rights reserved. 


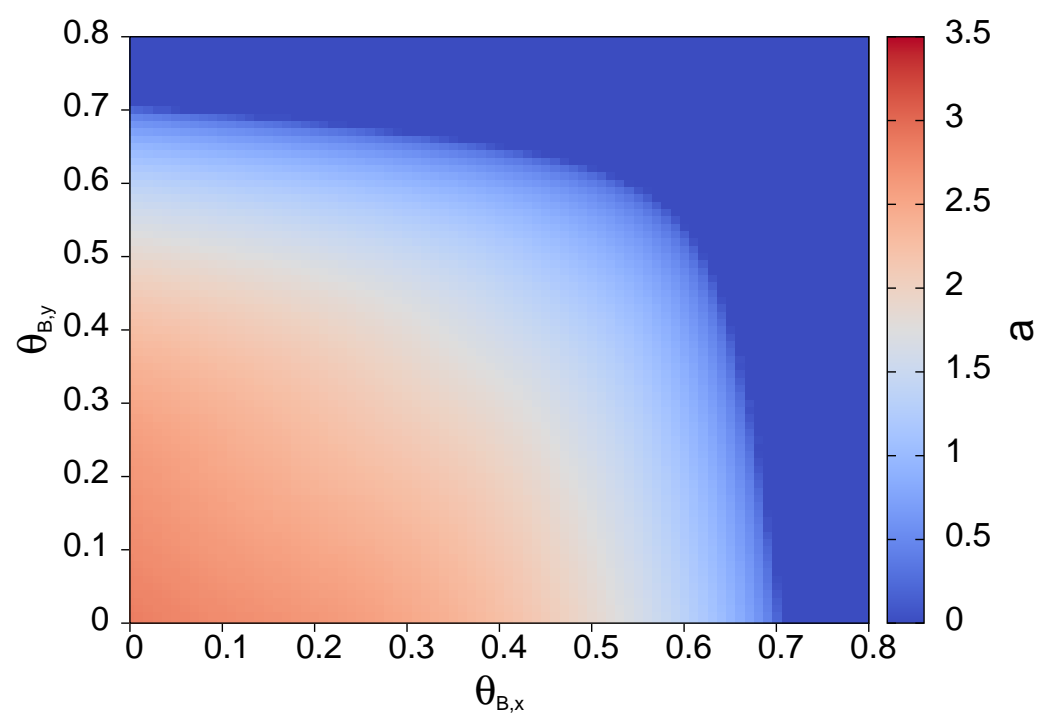

Figure 12. (Colour online) Amplitude, $a$, of the ground state thermal solitary wave as a function of $\left(\theta_{B, x}, \theta_{B, y}\right)$. The other parameters are $\beta_{x}=\beta_{y}=100$.
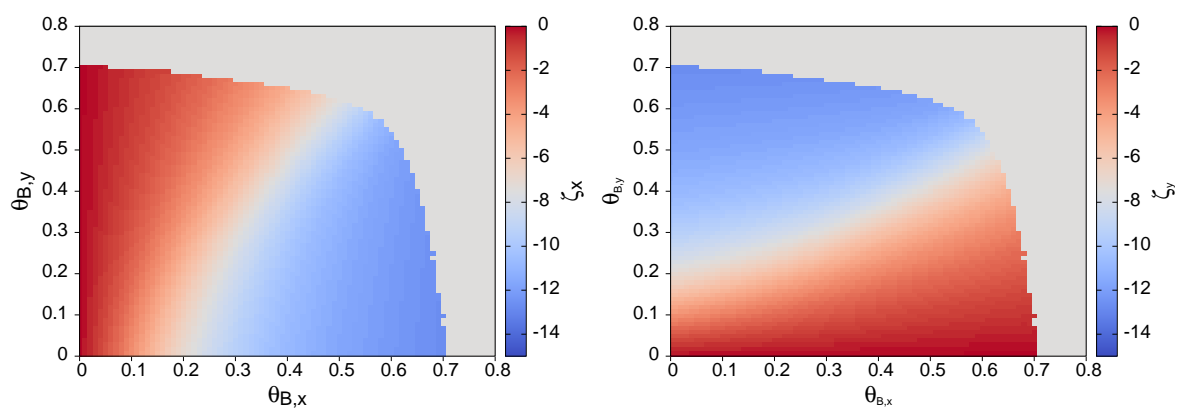

Figure 13. (Colour online) Position of the ground state thermal solitary wave for values in $\left(\theta_{B, x}, \theta_{B, y}\right)$ parameter space. a) shows offset along the $x$ axis, $\zeta_{x}$ and $\mathrm{b}$ ) shows offset along the $y$ axis $\zeta_{y}$. The other parameters are $\beta_{x}=\beta_{y}=100$. Location is not shown for $a<10^{-2}$. 


\subsection{Vakhitov-Kolokolov stability Criterion}

The stability of the thermal solitary waves will now be investigated, both analytically and numerically. The self-focussing mechanism for these solitary waves derives from the increase of the refractive index of the medium with the temperature increase due to the optical heating. This relationship is analogous to the optical Kerr effect and can result in modulational instability of a perturbed propagating solitary wave. To investigate this, power versus propagation constant curves are calculated for the families of thermal solitary waves given by (10). The optical power

$$
P=\int_{-L / 2}^{+L / 2} \int_{-L / 2}^{L / 2}|E|^{2} d x d y
$$

is calculated using the numerical solitary wave solutions found using the Newton-CG method. By the Vakhitov-Kolokolov (VK) stability criterion [1] it is a necessary, but not sufficient, condition for the modulational stability of solitary wave solutions of generalised NLS equations on an infinite domain that the solution falls within a region of parameter space for which the power versus propagation constant curve has positive slope $(d P / d \mu>0)$, see, for example, [39, 40,41]. This result has not been theoretically proven for an NLS-type system on a finite domain, but the results presented here are consistent with this infinite domain theory and with the $(1+1)-\mathrm{D}$ case [29].

Figure 14(a) shows the power $P$ versus propagation constant $\mu$ for ground state thermal solitary waves. The three curves correspond to $\theta_{B, y}=0,0.2$ and 0.4 . For non-zero ambient temperature $\theta_{B, y}$ the solitary wave solution branch only exists for a finite range of $\mu$. All three curves are monotonic with positive slope indicating that, for a given value of the ambient temperature, there is a single stable solution branch. Figure 14(b) shows the power $P$ versus propagation constant $\mu$ for ground and excited state thermal solitary waves for $\theta_{B, y}=0.2$. The three curves correspond to the ground state and the first two excited states. In all three cases the curves are monotonic with positive slope, fulfilling the necessary condition for modulational stability across the entire range of $\mu$ values for both the ground and excited state solitary waves.

This article is protected by copyright. All rights reserved. 

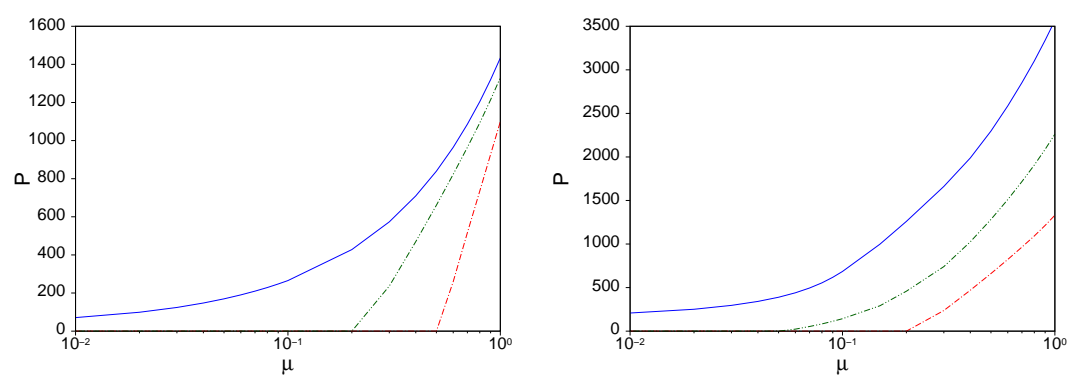

Figure 14. (Colour online) (a) Power $P$ versus propagation constant $\mu$, for ground state thermal solitary waves. Shown are $\theta_{B, y}=0$ : blue (solid) line; $\theta_{B, y}=0.2$ : green (dash-dot-dot) line; $\theta_{B, y}=0.4$ : red (dashdot) line. (b) Power $P$ versus propagation constant, $\mu$, for ground and excited state thermal solitary waves for $\theta_{B, y}=0.2$. Ground state wave: red (dash-dot) line; first excited state: green (dash-dot-dot) line; second excited state: blue (solid) line.

\subsection{Spectral stability analysis}

In Section 4.1 the V.K. condition, a necessary condition for modulational stability of thermal solitary waves, was examined. However, it is still possible that amplitude or drift instabilities may occur, see [36]. To assess this possibility, we shall now use a linearized stability analysis following the technique outlined in [35]. Instabilities occur if there are negative eigenvalues. In this analysis a perturbation to the steady state solution is expressed as a superposition of linear modes. Note that we are not considering perturbations resulting from the boundary conditions. We hence express $u$ and $\theta$ as

$$
\begin{aligned}
& u=u_{s}+\epsilon\left[(v-w) e^{\imath \lambda z}+\left(v^{*}+w^{*}\right) e^{-\imath \lambda^{*} z}\right] e^{\imath \mu z} \\
& \theta=\theta_{s}+\epsilon \Theta\left[e^{\imath \lambda z}+e^{-\imath \lambda^{*} z}\right]
\end{aligned}
$$

where $u_{s}$ and $\theta_{s}$ are the steady state solutions and $\epsilon$ is a small parameter.

Substituting the perturbed solitary wave solution (15) into the electric field equation of (4), we obtain

$$
(-\mu-\lambda)(w-v)+\frac{1}{2}\left(\nabla^{2} v-\nabla^{2} w\right)+2 \theta_{s}(v-w)+2 u_{s} \Theta=0 .
$$

This article is protected by copyright. All rights reserved. 
To set this into an appropriate form for an eigenvalue problem, we need to eliminate the temperature perturbation $\Theta$. Substituting the perturbation expansions for $u$ and $\theta$ into the temperature equation of (11) gives

$$
\nu\left(\nabla^{2} \Theta e^{\imath \lambda z}+\nabla^{2} \Theta e^{-\imath \lambda^{*} z}\right)+4 u_{s}\left(u e^{\imath \lambda z}+u^{*} e^{-\imath \lambda^{*} z}\right)=0 .
$$

Taking the coefficients of $e^{\imath \lambda z}$ from (17) and solving for $\Theta$ gives

$$
\nu \nabla^{2} \Theta+4 u_{s} u=0
$$

which implies

$$
\Theta=\left[\nabla^{2}\right]^{-1} \frac{4 u_{s} u}{\nu} .
$$

Note that the Newton-cooling boundary conditions (8) and (9) have been applied in the inversion. Then substituting (19) back into (16) and converting these to the form of the eigenvalue problems $L_{0} v=\lambda w$ and $L_{1} w=\lambda v$, we find

$$
L_{0}=\left(\mu-\frac{1}{2} \nabla^{2}-2 \theta_{s}-\frac{8 u_{s}^{2}}{\nu}\left[\nabla^{2}\right]^{-1}\right), \quad L_{1}=\left(\mu-\frac{1}{2} \nabla^{2}-2 \theta_{s}\right),
$$

or

$$
L_{0} L_{1} w=\lambda^{2} w .
$$

We require the eigenvalues, $\lambda^{2}$, to be all positive for stability. The eigenvalues of $L_{0} L_{1}$ were calculated for a range of parameters. We found that the matrix $L_{0} L_{1}$ typically had a condition number of $O\left(10^{10}\right)$, indicating that the eigenvalue problem is very ill-conditioned. Standard single and double float variable types can only provide around 7 or 16 decimal digits of precision, respectively. Rounding errors introduced in the calculations mean that these precisions were found not to be sufficient to calculate all minimum eigenvalues accurately. In many cases, the error in the computed minimum eigenvalue is sufficient that its sign would be incorrect, leading to an incorrect inference about the stability of the solitary wave system. To determine a suitable numerical precision to use for calculating the minimum eigenvalues, a number of cases were tested using the BigFloat type in the Julia language, which is based on the GNU MFPR library (multiple-precision binary floating-point library with correct rounding) [42].

Figure 15 shows the minimum eigenvalue, $\log \left(\left|\lambda^{2}\right|\right)$, versus numerical floating point precision for two cases, $\mu=0.5$ and 1 . The other parameter is $\theta_{B, y}=0$. Note that in Figures $15-17$, which show minimum 
eigenvalues, the plots are constructed to display the negative and positive eigenvalues on a log scale by first taking the absolute value. The upper panel in each plot shows the region where the minimum eigenvalue $\lambda^{2}$ is positive, while the bottom panel shows the region where $\lambda^{2}$ is negative. There is a discontinuity on the $y$ axis, given by a solid line, representing the transition from negative to positive values.

The case $\mu=1$ has a negative minimum eigenvalue for all precisions, converging to a value of $\lambda^{2}=-4.9319 \times 10^{-2}$. In this case the solution converged to a consistent result with 5 significant digits with a numerical precision of 64 bits. For the case $\mu=0.5$ the standard single precision of 32 bits resulted in an incorrect sign of the computed minimum eigenvalue, with convergence to the value $\lambda^{2}=1.08975 \times 10^{-8}$ to within 5 significant digits occurring at a numerical precision of 64 bits. Based on these trials, the minimum eigenvalues were calculated using numerically extended precision to 128 bits to ensure that rounding errors did not have a significant impact. The use of extended precision added significantly to the computation costs for these calculations, as a result of the extra overheads inherent in these variable types in programming languages.

Figure 16(a) shows the minimum eigenvalue, $\log \left(\left|\lambda^{2}\right|\right)$ versus $\theta_{B, y}$, for (21). Shown are $\mu=0.5,0.7$ and 1. Figure 16(b) shows amplitude $a$ versus $\theta_{B, y}$, with stable region shown as solid line and the unstable region shown as dashed lines. The symbols $A, B, C$ and $D$ refer to the parameter choices used for Figures 18, 19, 20 and 21, respectively. In the case $\mu=1.0$, the smallest eigenvalue is negative for values of $\theta_{B, y}$ up to 0.175 , after which all the eigenvalues are positive. For the case $\mu=0.5$, the eigenvalues are positive for all values of $\theta_{B, y}$ up to 0.5 , suggesting stability for $\theta_{B, y}$ in this range. For $\mu=0.7$ the threshold value for the sign change of the minimum eigenvalue is $\theta_{B, y}=0.075$, with stability for large values of $\theta_{B, y}$. The figures shows that higher amplitude solitary waves are generally unstable while lower amplitude waves are generally stable. Increasing $\theta_{B, y}$ stabilizes the solitary waves, as the amplitudes are lower for off-centred waves, while increasing $\mu$ destabilizes the waves, as their amplitude increases.

Figure 17(a) shows the minimum eigenvalue $\log \left(\left|\lambda^{2}\right|\right)$ versus $\mu$ for (21). Figure 17(b) shows the amplitude versus $\mu$ for (21). Shown are $\theta_{B, y}=0.0,0.05$ and 0.1 . The symbols $A, B, C$ and $D$ refer to the parameter choices used for Figures 18, 19, 20 and 21, respectively. The solitary wave is not stable for larger values of the propagation constant,

This article is protected by copyright. All rights reserved. 


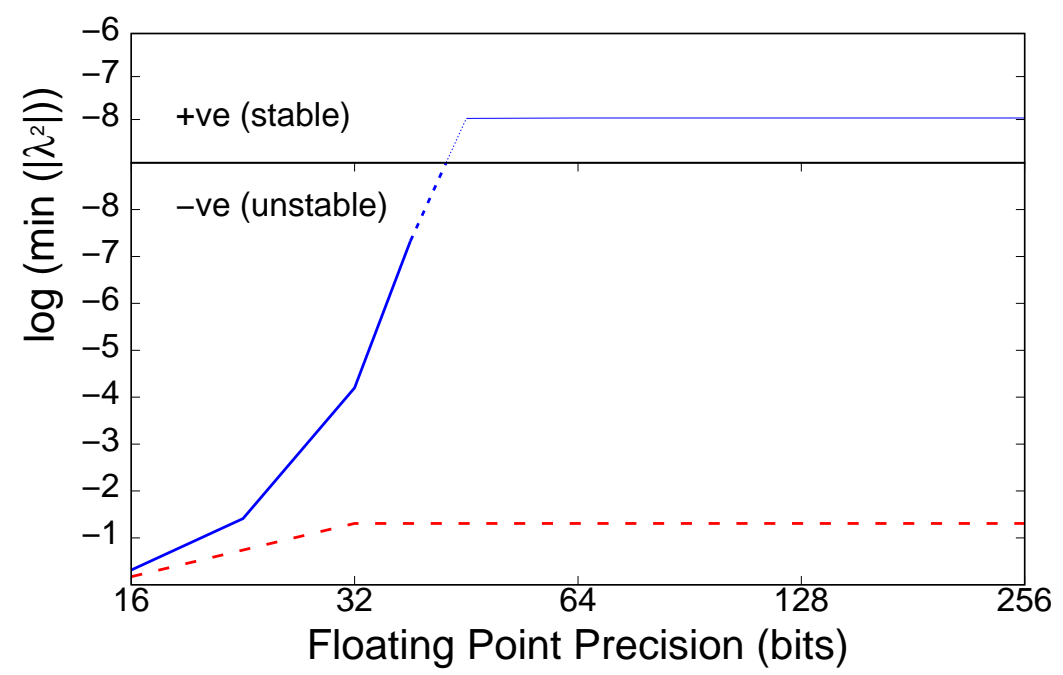

Figure 15. (Colour online) Minimum eigenvalue, plotted as $\log \left(\left|\lambda^{2}\right|\right)$, versus numerical floating point precision for ground state thermal solitary waves. The parameters are $\theta_{B, y}=0$ and $\mu=1$ : red (dashed) line; $\mu=0.5$ : blue (solid) line.

indicating that regions of parameter space corresponding to large amplitude solitary waves are unstable, according to the linearized stability analysis. To assess whether the negative eigenvalue is associated with an amplitude stability or a drift instability it is necessary to check whether the associated eigenmode is symmetric or asymmetric [36]. In this case, the negative eigenvalues possess a symmetric eigenmode, indicating that for values of $\mu>0.55$ for the $\theta_{B, y}=0$ case the solitary wave possesses an amplitude instability. For values of $\mu<0.55$, the solitary wave is stable.

\subsection{Numerical Simulations}

The analytical stability predictions will now be compared with the results of numerical simulations of the governing thermal system (4) to (9),

This article is protected by copyright. All rights reserved. 

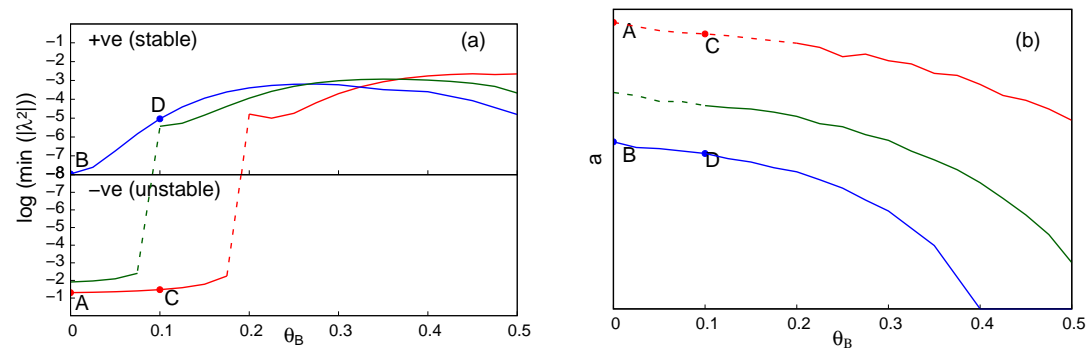

Figure 16. (Colour online) (a) Minimum eigenvalue $\log \left(\left|\lambda^{2}\right|\right)$ versus $\theta_{B, y}$ for (21). Shown are $\mu=1.0$ : red (right) line; $\mu=0.7$ : green (middle) line; $\mu=0.5$ : blue (left) line. The dashed lines shows the discontinuity where the minimum eigenvalue changes sign. (b) Amplitude $a$ versus $\theta_{B, y}$, with stable region shown as solid line and the unstable region shown as dashed lines. Shown are $\mu=1.0$ : red (top) line; $\mu=0.7$ : green (middle) line; $\mu=0.5$ : blue (bottom) line.
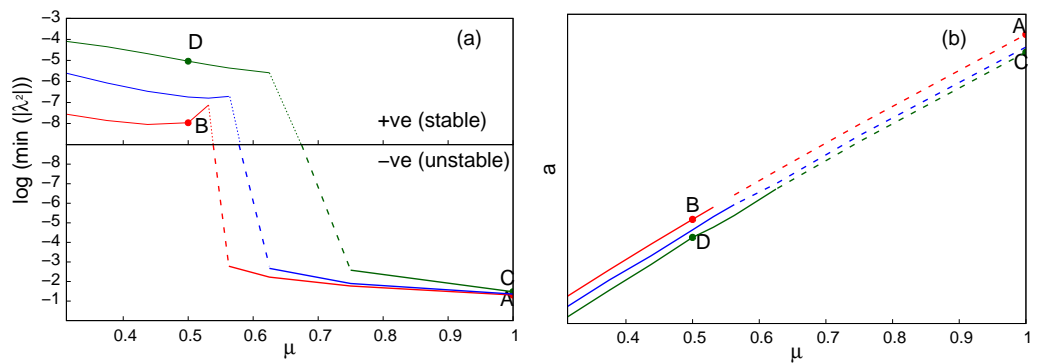

Figure 17. (Colour online) (a) Minimum eigenvalue $\log \left(\left|\lambda^{2}\right|\right)$ versus $\mu$ for (21). Shown are $\theta_{B, y}=0.0$ : red (left) line; $\theta_{B, y}=0.05$ : blue (middle) line; $\theta_{B, y}=0.1$ : green (right) line. (b) Amplitude $a$ versus $\mu$, with stable region shown as solid line and the unstable region shown as dashed lines. Shown are $\theta_{B}=0.0$ : red (top) line; $\theta_{B}=0.05$ : blue (middle) line; $\theta_{B}=0.1$ : green (bottom) line.

This article is protected by copyright. All rights reserved. 
using the numerical steady state solution as an initial condition. The initial condition used is then

$$
E(x, y, 0)=u_{s}(x, y)+\epsilon_{0} \times \phi(x, y), \quad \theta(x, y, 0)=\theta_{s}(x, y),
$$

where $u_{s}(x, y)$ and $\theta_{s}(x, y)$ are the thermal solitary wave solution found using the Newton-CG method. The $\phi$ function generates a random normally distributed value between 0 and 1 for all $x$ and $y$. For the examples considered below, $\epsilon_{0}$ is taken as 0.01 , or about $0.3 \%$ of the amplitude of the solitary wave. The numerical solution of the electric field equation is obtained using a hybrid numerical method, where the spatial derivatives are discretized using central central differences and the fourth order Runge-Kutta method is used to advance in the time-like variable $z$. Gauss-Seidel iteration is used to solve for the temperature at each $z$-step [43]. The spatial discretizations used are $\Delta z=0.03$ and $\Delta x=\Delta y=0.682$.

Figure 18 shows the electric field $|E|$ and temperature $\theta$ in the $(y, z)$ plane, as given by the full numerical solution of the thermal system (4) with boundary conditions (6) to (9), with the profile taken along the $x=0$ axis. Shown is the numerical evolution of a perturbed solitary wave for $z$ up to 3000 . The parameters are $\theta_{B, y}=0.0$ and $\mu=1$. The initial thermal solitary wave corresponds to the symmetric case in Figure 3 . The thermal solitary wave is initially a ground state beam centred on $(x, y)=(0,0)$ with an amplitude of $a=2.86$. Instability develops from around $z=2000$, before the numerical solution breaks down completely after $z=2500$. This example corresponds to the parameter choices A marked on Figures 16 and 17, so the spectral stability analysis in this case has a negative minimum eigenvalue, which implies instability. The theoretical prediction of instability is then consistent with numerical solutions. It can be seen that starting at round $z=2000$ there is significant reflection from the boundaries, with the beam dropping in intensity. These reflections grow and eventually strongly interact with the main beam, resulting in it falling apart.

Figure 19 shows the electric field $|E|$ and temperature $\theta$ in the $(y, z)$ plane as given by the full numerical solution of the thermal equations (4) with boundary conditions (6) to (9), with the profile taken along the $x=0$ axis. Shown is the numerical evolution of the perturbed solitary wave for $z$ up to 6000 . The parameters are $\theta_{B, y}=0.0$ and $\mu=0.5$. In this case the initial thermal solitary wave is a ground state beam centred at $(x, y)=(0,0)$ with an amplitude of $a=1.68$. The 

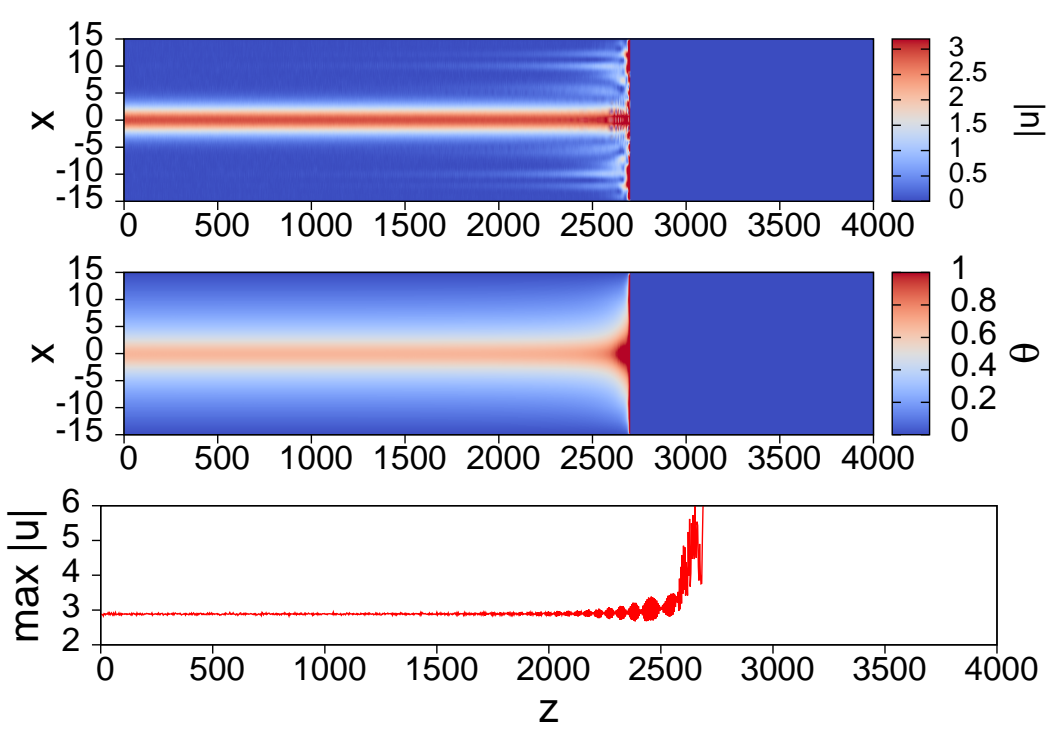

Figure 18. (Colour online) Time evolution of centred thermal solitary wave demonstrating instability. Shown are $u$ (top) and $\theta$ (middle), both taken along $y=0$, along with the maximum value of $u$ (bottom) from $z=0$ to $z=4000$. The parameters are $\theta_{B, y}=0$ and $\mu=1.0$.

solitary wave remains numerically stable until at least $z=6000$. This example corresponds to the parameter choices $B$ marked on Figures 16 and 17, so the spectral stability analysis in this case has a positive minimum eigenvalue, which implies stability. The theoretical prediction of stability is then again consistent with numerical solutions.

Figure 20 shows the electric field $|E|$ and temperature $\theta$ in the $(y, z)$ plane as given by the full numerical solution of the thermal equations (4) with boundary conditions (6) to (9). The other parameters are $\theta_{B, y}=0.1$ and $\mu=1.0$. The thermal solitary wave solution is a ground state beam off-centred at $(x, y)=(0,-3)$ with an amplitude $a=2.7$. Instability develops from around $z=600$, before the numerical solution breaks down completely after $z=800$. This example corresponds to the parameter choices $C$ marked on Figures 16 and 17, so the spectral stability analysis in this case has a negative minimum eigenvalue, which implies instability, again consistent with numerical solutions. The 

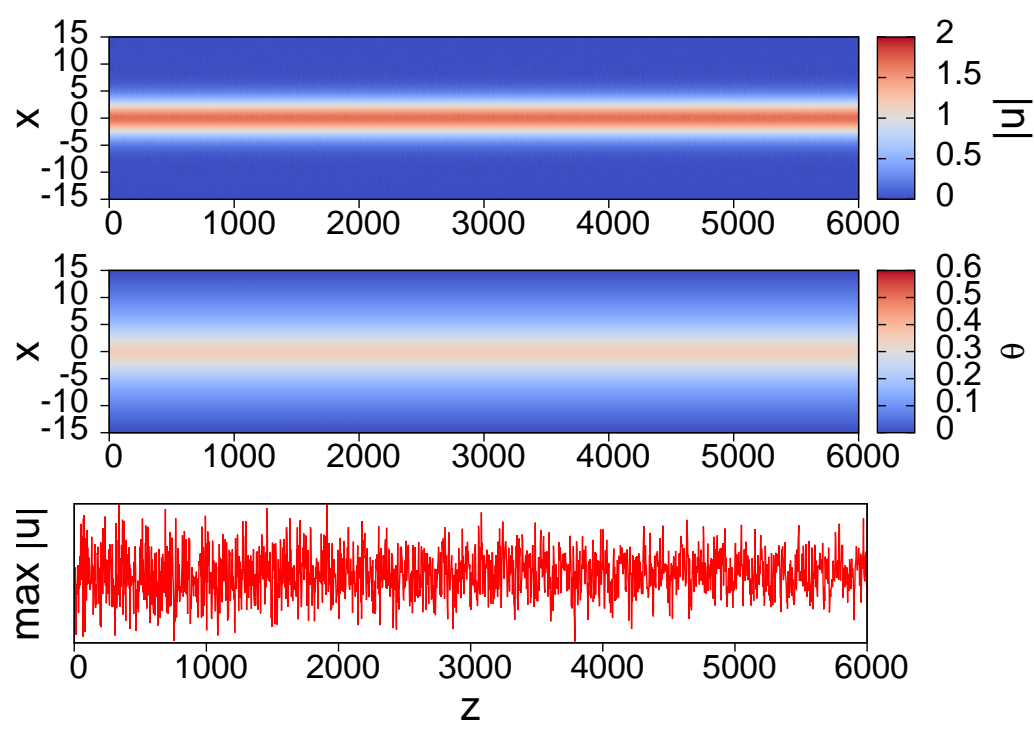

Figure 19. (Colour online) Time evolution of centred thermal solitary wave, demonstrating stability of the solution. Shown are $u$ (top) and $\theta$ (middle), both taken along $y=0$. Maximum value of $u$ (bottom) from $z=0$ to $z=6000$. The parameters are $\theta_{B, y}=0$ and $\mu=0.5$.

instability mechanism is similar to that for the beam displayed in Figure 18, only stronger. There are growing reflections from the boundaries, starting at $z=600$, which interact with the beam with increasing strength until the beam is deflected and is destroyed.

Figure 21 shows the electric field $|E|$ and temperature $\theta$ in the $(y, z)$ plane as given by the full numerical solution of the thermal equations (4) with boundary conditions (6) to (9). The parameters are $\theta_{B, y}=0.1$ and $\mu=0.5$. The thermal solitary wave solution is a ground state beam off-centred at $(x, y)=(0,-6.18)$ with an amplitude $a=1.55$. Here, the beam is much closer to the $y=-15$ boundary than for the example considered in Figure 20. For this case, no instability develops by $z=2000$, although it is seems from the plot of $\max |u|$ that instability is beginning to develop by $z=2500$ for this off-centred case. This example corresponds to the parameter choices $D$ marked on Figures 16 and 17, so the spectral stability analysis in this case has a positive minimum

This article is protected by copyright. All rights reserved. 
eigenvalue, which implies stability. The correspondence between the theoretical stability prediction and numerical solutions is then not clearcut. Whilst this example exhibits numerical stability for a much longer $z \approx 3000$ than does Figure 20 (which exhibits instability at $z \approx 700$ ), numerical instability does eventually develop. This instability may be numerical and due to the challenges of propagating an off-centred wave, or perhaps due to influence from the boundaries which are not captured in our stability analysis.
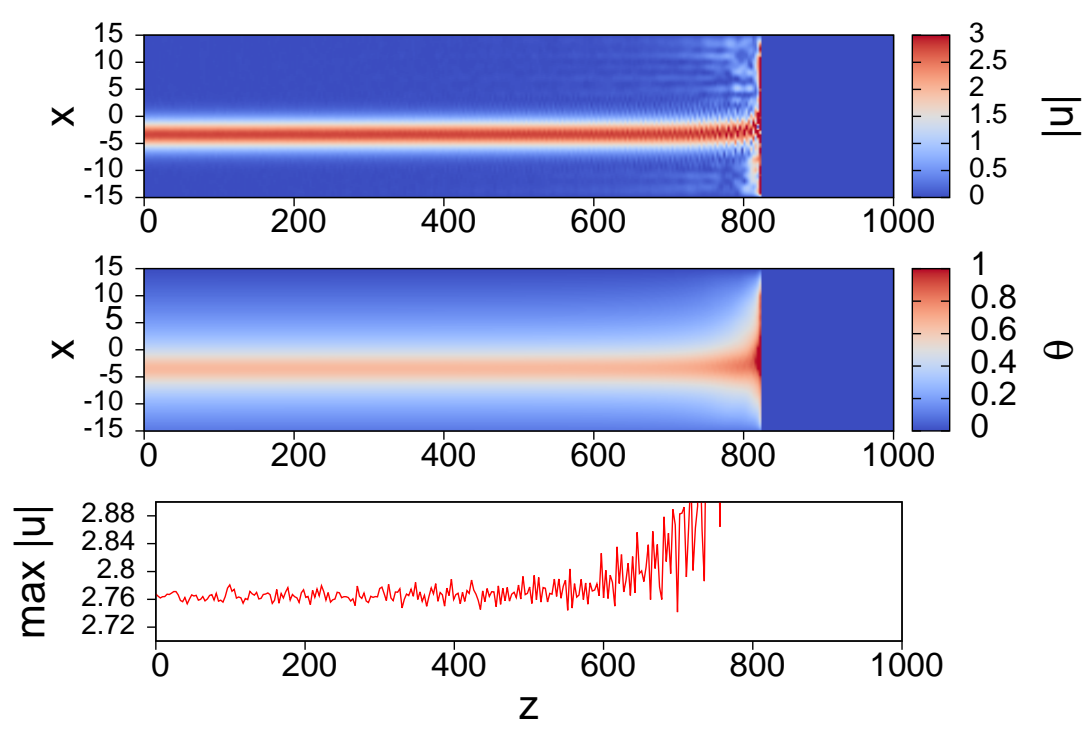

Figure 20. (Colour online) Time evolution of off-centre thermal solitary wave, demonstrating instability of the solution. Shown are $u$ (top) and $\theta$ (middle), both taken along $y=0$. Maximum value of $u$ (bottom) from $z=0$ to $z=2000$. The parameters are $\theta_{B, y}=0.1$ and $\mu=1.0$.

\section{Conclusions}

We have demonstrated that the Newton-CG method can be used to find steady state solutions for $(2+1)$-D solitary waves propagating in thermal media, including cases for which one of more of the cell boundaries is

This article is protected by copyright. All rights reserved. 

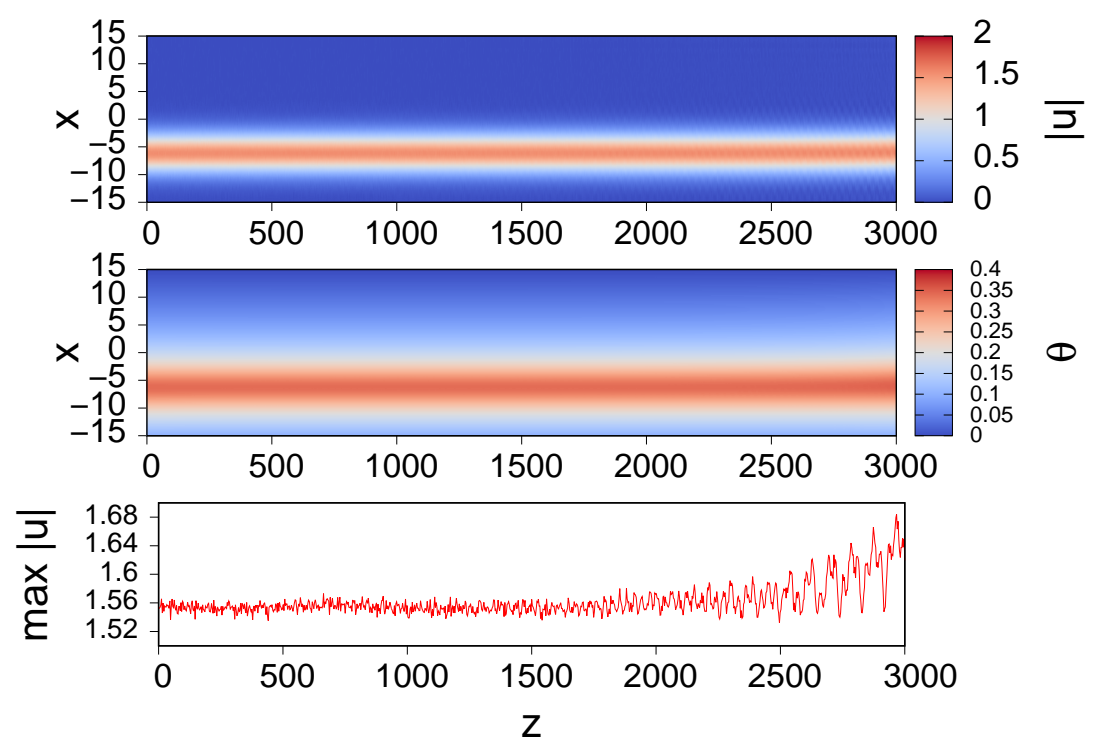

Figure 21. (Colour online) Time evolution of off-centre thermal solitary wave, demonstrating stability of the solution. Shown are $u$ (top) and $\theta$ (middle), both taken along $y=0$. Maximum value of $u$ (bottom) from $z=0$ to $z=3000$. The parameters are $\theta_{B, y}=0.1$ and $\mu=0.5$.

warmer than the ambient temperature. The solutions found here show that the location of an optical beam within a cell migrates towards the warmer boundaries and they can be guided towards the corner of the cell when two adjacent boundaries are warmer than the ambient temperature. The results of the analytical stability analysis were found to be in agreement with numerical solutions. It was shown both analytically and numerically that the thermal solitary waves are stable for combinations of boundary temperature and propagation constant corresponding to solitary waves of lower amplitude. These results may have direct application for experiments into the control and steering of light beams in optical media.

Acknowledgement: The authors wish to thank an anonymous referee for their helpful suggestions.

This article is protected by copyright. All rights reserved. 


\section{Appendix A: The Newton-Conjugate Gradient method}

To describe the Newton-CG method, consider (10) for a steady solitary wave rewritten in the form

$$
L_{00} v(x, y)=\mu v(x, y), \text { where } L_{00} \equiv \frac{1}{2} \frac{\partial^{2}}{\partial x^{2}}+\frac{1}{2} \frac{\partial^{2}}{\partial y^{2}}+2 \theta .
$$

where $L, L_{0}, L_{00}$ and $M$ are all operators, which correspond to matrices once finite difference approximations for the spatial second derivative terms are applied. To implement the Newton-CG method, we consider (A1) in the form $L_{0} v(x, y)=0$, where $L_{0}=L_{00}-\mu$. Given an approximate solution $v_{n}$, we can write $v=v_{n}+e_{n}$, where $v$ is the exact solution and $e_{n}$ is the error. Substituting this into (A1) and neglecting higher-order terms in $e$, we obtain

$$
L_{1 n} \Delta v_{n}=-L_{0} v_{n}
$$

where the next approximation is $v_{n+1}=v_{n}+\Delta v_{n}$ and $L_{1 n}$ is the linearisation operator evaluated at the iterate $v_{n}$. Equation (A2) is solved for $\Delta v_{n}$ using the standard conjugate gradient method. In general, the convergence of the conjugate gradient method requires that the matrix $L_{1 n}$ be self-adjoint, which is not the case here. To circumvent this, we pre-multiply both sides of (A2) by $L_{1 n}^{T}$ prior to solving. This has the effect of greatly increasing the condition number of the matrix $\left(L_{1 n}^{T} L_{1 n}\right)$ and also the computation time, but does not change the solution, and ensures that the method converges.

Selecting a suitable discretization scheme for the iterative methods requires some care, as there is limited benefit in setting the stopping condition for the scheme at a lower level than the error introduced by the discretization. In all of the following discussion we assume that $\Delta x=\Delta y$, and just refer to $\Delta x$ to indicate the discretization grid size. The amplitude of the solitary wave can be expanded in the series

$$
a=a(\Delta x)+a_{1} \Delta x^{4}+O\left(\Delta x^{6}\right),
$$

as the fourth order Runge-Kutta method is used, where $a$ is the exact solution, $a(\Delta x)$ is the numerically derived solution and $a_{1}$ the amplitude of the fourth-order error term. Taking $\Delta x=0.5$ and $\Delta x=1.0$ with $\mu=1, \beta_{y}=100$ and $\theta_{B, y}=0$ yields the values $a(0.5)=2.854377$ and $a(1.0)=2.880987$, respectively. We can then derive higher order approximations to $a$ and the leading order error $a_{1}$ on using Richardson

This article is protected by copyright. All rights reserved. 
extrapolation, as

$$
\begin{gathered}
a=\frac{2^{4} a(0.5)-a(1.0)}{2^{4}-1}+O\left(\Delta x^{5}\right) \approx 0.2 .852603 \\
a_{1}=\frac{a(0.5)-a(1.0)}{(1.0)^{4}\left(1-\frac{1.0}{2^{4}}\right)}+O\left(\Delta x^{5}\right) \approx-2.84 \times 10^{-2} .
\end{gathered}
$$

From these we can calculate that the discretization error when $\Delta x=0.5$ is $a-a(0.5) \approx 1.77 \times 10^{-3}$. The stopping condition $\epsilon$ should then be less than $\mathrm{O}\left(10^{-3}\right)$ and greater than the round-off error. Hence, we choose $\epsilon=10^{-10}$, which is suitable for the choice $\Delta x=0.5$ and also for much smaller choices of $\Delta x$.

\section{References}

1. Y. Kivshar and G. Agrawal, Optical Solitons: From Fibers to Photonic Crystals, San Diego Academic, 2003.

2. M. Peccianti and G. Assanto, Nematicons, Phys. Reports 516:147-208 (2012).

3. A. B. Aceves, J. V. Moloney, and A. C. Newell, Theory of light-beam propagation at nonlinear interfaces. I Equivalent-particle theory for a single interface, Phys. Rev. A 39:1809-1827 (1989).

4. C. Conti, M. Peccianti, and G. Assanto, Route to nonlocality and observation of accessible solitons, Phys. Rev. Lett. 91 (2003), no. 073901.

5. F. W. DabBy and J. R. Whinnery, Thermal self-focusing of laser beams in lead glasses, Appl. Phys. Lett. 13:284-286 (1968).

6. C. Rotschild, O. Cohen, O. Manela, M. Segev, and T. Carmon, Solitons in nonlinear media with an infinite range of nonlocality: First observation of coherent elliptic solutions and vortex ring solutions, Phys. Rev. Lett. 95 (2005), no. 213904 .

7. E. Kuznetsov, A. Rubenchik, and V. Zakharov, Soliton stability in plasmas and hydrodynamics, Phys. Rep. 142:103-165 (1986).

8. M. Segev, B. Crosignani, A. Yariv, and B. Fischer, Spatial solitons in photorefractive media, Phys. Rev. Lett. 68:923-926 (1992).

9. C. Conti and G. Assanto, Nonlinear Optics Applications: Bright Spatial Solitons, vol. 5, Elsevier, Oxford, 2004.

10. A. G. Litvak, Self-focusing of powerful light beams by thermal effects, JETP Lett. 4:230-233 (1966).

11. A. A. Minzoni, N. F. Smyth, and A. L. Worthy, Modulation solutions for nematicon propagation in nonlocal liquid crystals, J. Opt. Soc. Am. B 24:15491556 (2007).

This article is protected by copyright. All rights reserved. 
12. A. Minovich, D. Neshev, A. Dreischuh, W. Krolikowski, and Y. Kivshar, Experimental reconstruction of nonlocal response of thermal nonlinear optical media, Opt. Lett. 32:1599-1601 (2007).

13. C. Barsi, W. Wan, C. Sun, and J. Fleischer, Dispersive shock waves with nonlocal nonlinearity, Opt. Lett. 32:2930-2932 (2007).

14. G. A. El and M. A. Hoefer, Dispersive shock waves and modulation theory, Physica D 333:11-65 (2016).

15. C. Conti, A. Fratalocchi, M. Peccianti, G. Ruocco, and S. Trillo, Observation of a gradient catastrophe generating solitons, Phys. Rev. Lett. 102 (2009), no. 083902.

16. B. Alfassi, C. Rotschild, O. Manela, M. Segev, and D. Christodoulides, Boundary force effects exerted on solitons in highly nonlocal media, Opt. Lett. 32:154-156 (2007).

17. C. HuAng, Propagation of coupled dipole-mode beams in nonlocal thermal media, Opt. Commun. 284:5786-5791 (2011).

18. M. Peccianti, K. A. Brzakiewicz, and G. Assanto, Nonlocal spatial soliton interactions in nematic liquid crystals, Opt. Lett. 27:1460-1462 (20002).

19. A. Fratalocchi, M. Peccianti, C. Conti, and G. Assanto, Spiralling and cyclic dynamics of nematicons, Mol. Cryst. Liq. Cryst. 421:197-207 (2004).

20. J. M. L. MacNeil, N. F. Sмyth, and G. Assanto, Exact and approximate solutions for solitary waves in nematic liquid crystals, Physica D 284:1-15 (2014).

21. B. Malomed, Variational methods in nonlinear fiber optics and related fields, Prog. Opt. 43:71-193 (2002).

22. T. R. Marchant and N. F. Smyth, Nonlocal validity of an asymptotic one dimensional nematicon solution, J. Phys A.:Math. Theor. 41 (2008), no. 365201.

23. T. R. Marchant and N. F. Smyth, Solitary waves and their stability in colloidal media: Semi-analytical solutions, Dyn. Cont. Disc. Impul. Sys. 19:525-541 (2012).

24. F. A. Sala, N. F. Smyth, U. A. Laudyn, M. A. Karpierz, A. A. Minzoni, and G. Assanto, Bending reorientational solitons with modulated alignment, J. Opt. Soc. Amer. B 34:2459-2466 (2017).

25. A. A. Minzoni, N. F. Smyth, and G. Assanto, Light self-localization in nematic liquid crystals: modelling solitons in nonlocal reorientational media, J. Nonlin. Opt. Phys. Mat. 18:657-691 (2009).

26. G. Assanto, A. A. Minoni, M. Peccianti, and N. F. Smyth, Optical solitary waves escaping a wide trapping potential in nematic liquidcrystals: modulation theory, Phys. Rev. A 79:033837 (2009).

27. U. A. Laudyn, M. Kwaśny, F. A. Sala, M. A. Karpierz, N. F. Smyth, and G. Assanto, Curved optical solitons subject to transverse acceleration in reorientational soft matter, Nature Sci. Reports 7:12385 (2017).

28. P. D. Rasmussen, O. Bang, and W. Krolikowski, Theory of nonlocal soliton interaction in nematic liquid crystals, Phys. Rev. E 72 (2005), no. 066611.

29. S. A. Louis, T. R. Marchant, and N. F. Sмyth, Optical solitary waves in thermal media with non-symmetric boundary conditions, J. Phys. A.:Math. Theor. 46 (2013), no. 055201.

This article is protected by copyright. All rights reserved. 
30. J. YANG, Newton-conjugate-gradient methods for solitary wave computations, J. Comp. Phys. 228:7007-7024 (2009).

31. J. YANG and T. I. LAKOBA, Accelerated imaginary-time evolution methods for the computation of solitary waves, Stud. Appl. Math. 120:265-292 (2008).

32. J. YANG and T. I. LAKOBA, Universally-convergent squared operator iteration methods for solitary waves in general nonlinear wave equations, Stud. Appl. Math. 118:153-197 (2007).

33. H. WANG, Numerical studies on the split-step finite difference method for nonlinear Schrodinger equations, App. Math. Comp. 170 (2005).

34. D. Suter and T. Blasberg, Stabilization of transverse solitary waves by a nonlocal response of the nonlinear medium, Phys. Rev. A 48:4583-4588 (1993).

35. A. Yakimenko, Y. Zaliznyak, and Y. S. Kivshar, Stable vortex solitons in nonlocal self-focusing nonlinear media, Phys. Rev. E 71 (2005), no. 065603.

36. B. Ilan, Y. Sivan, and G. Fibich, A quantitative approach to soliton instability, Opt. Lett. 36:397-399 (2011).

37. A. Alberucci, M. Peccianti, and G. Assanto, Nonlinear bouncing of nonlocal spatial solitons at the boundaries, Opt. Lett. 32:2795-2797 (2007).

38. A. Alberucci and G. Assanto, Propagation of optical spatial solitons in finitesize media: interplay between nonlocality and boundary conditions, J. Opt. Soc. Am. B 24:2314-2320 (2007).

39. R. H. Enns and S. S. Rangnekar, Bistable-soliton pulse propagation, Phys. Rev. A 36 (1987), no. 3.

40. A. E. Kaplan, Bistable solitons, Phys. Rev. Lett. 55:1291-1294 (1985).

41. R. H. Enns, S. S. Rangnekar, and A. E. Kaplan, Robust bistable solitons of the highly nonlinear Schrodinger equation, Phys. Rev. A. 35:466-469 (1987).

42. L. Fousse, G. Hanrot, V. Lefevre, P. Pelissier, and P. Zimmermann, MPFR: A multiple precision binary floating-point library with correct rounding, ACM Trans. Math. Softw. 33 (2007), no. 13.

43. G. Assanto, T. R. Marchant, A. A. Minzoni, and N. F. Smyth, Reorientational versus Kerr dark and grey solitary waves using modulation theory, Phys. Rev. E. 84 (2011), no. 066602.

\footnotetext{
ga11b1 School of Mathematics and Applied Statistics, The University of Wollongong, Wollongong, 2522, N.S.W., Australia

ga11в2 School of Mathematics, University of Edinburgh, Edinburgh EH9 3FD, Scotland, U.K.
}

This article is protected by copyright. All rights reserved. 


\section{University Library}

\section{- M M N E R VA A gateway to Melbourne's research publications}

Minerva Access is the Institutional Repository of The University of Melbourne

Author/s:

Louis, SA;Marchant, TR;Smyth, NF

Title:

2-D solitary waves in thermal media with nonsymmetric boundary conditions

Date:

2019-05-01

Citation:

Louis, S. A., Marchant, T. R. \& Smyth, N. F. (2019). 2-D solitary waves in thermal media with nonsymmetric boundary conditions. STUDIES IN APPLIED MATHEMATICS, 142 (4), pp.586-607. https://doi.org/10.1111/sapm.12243.

Persistent Link:

http://hdl.handle.net/11343/285155 